\title{
A versatile electrostatic trap
}

\author{
Jacqueline van Veldhoven ${ }^{1,2}$, Hendrick L. Bethlem ${ }^{1,3}$, Melanie Schnell ${ }^{1}$, and Gerard Meijer ${ }^{1}$ \\ ${ }^{1}$ Fritz-Haber-Institut der Max-Planck-Gesellschaft, Faradayweg 4-6, D-14195 Berlin, Germany \\ ${ }^{2}$ FOM-Institute for Plasmaphysics Rijnhuizen, P.O. Box 1207, NL-3430 BE Nieuwegein, The Netherlands \\ ${ }^{3}$ Laser Centre Vrije Universiteit Amsterdam, de Boelelaan 1081, NL-1081 HV Amsterdam, The Netherlands
}

(Dated: October 22, 2018)

\begin{abstract}
A four electrode electrostatic trap geometry is demonstrated that can be used to combine a dipole, quadrupole and hexapole field. A cold packet of ${ }^{15} \mathrm{ND}_{3}$ molecules is confined in both a purely quadrupolar and hexapolar trapping field and additionally, a dipole field is added to a hexapole field to create either a double-well or a donut-shaped trapping field. The profile of the ${ }^{15} \mathrm{ND}_{3}$ packet in each of these four trapping potentials is measured, and the dependence of the well-separation and barrier height of the double-well and donut potential on the hexapole and dipole term are discussed.
\end{abstract}

PACS numbers: 33.80.Ps, 33.55.Be, 39.10.+j

\section{INTRODUCTION}

The creation of cold molecules offers unique possibilities for studying cold collisions [1, 2, 3, 4], dipole-dipole interaction [5, 6, 7], or for doing high-resolution spectroscopy [8, 9]. In each of these fields, traps are either necessary, or they enhance the potential of the method considerably. Electrostatic traps in particular are highly versatile. They are rather deep (on the order of $1 \mathrm{~K}$ ) and by changing electrode geometries, many different trapping potentials can be formed 10, 11, 12, 13, 14, 15. Here we demonstrate an electrode geometry that is used to form different trapping fields by changing the voltage applied to the electrodes. In the trap a predominantly dipolar, quadrupolar or hexapolar field can be created. When implementing the latter two, a pure quadrupole or a pure hexapole trapping potential is generated. Combining a dipole field with a hexapole field gives rise to two additional trapping potentials, a double-well or a ringshaped potential, depending on the sign of the dipole term. As voltages can be switched on relatively short timescales, rapid changing between these trapping fields is feasible as well.

A double-well trap in particular has proven to be fruitful in atomic physics experiments. For instance, doublewell potentials have been used in interference experiments to study coherence properties of BEC's [16] and to visualize phase singularities associated with vortices in a Na BEC [17]. Furthermore, two weakly coupled BEC's in a double-well potential have been shown to form a Josephson junction [18], and collision studies have been performed in a double-well interferometer, enabling the determination of the $s$ - and $d$-wave scattering amplitudes of ${ }^{87} \mathrm{Rb}$ 19.

In electrostatic traps the formation of a double-well potential with variable barrier height and well separation is relatively straightforward. Along with the possibility of rapid switching to other types of trapping fields, this creates favorable conditions for the study of cold collisions. Molecules trapped in either of the two wells of a doublewell trap will gain potential energy when switching to a single-well trap, resulting in two packets of molecules

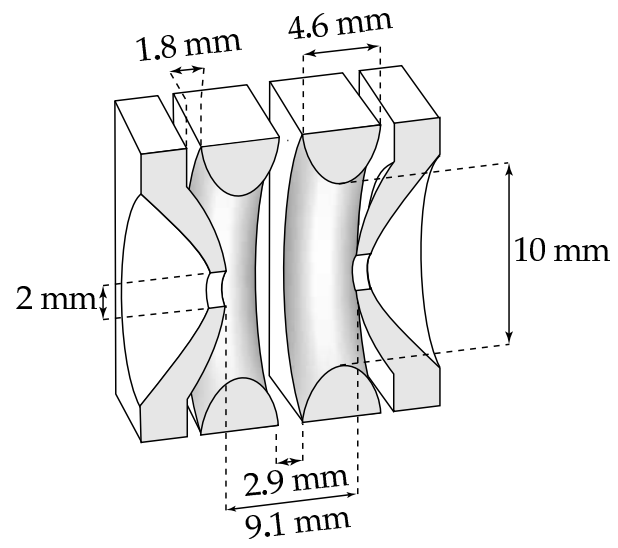

FIG. 1: Scheme of the trap. The trap is cylindrically symmetric and consists of two parallel rings in the middle and two endcaps at the sides.

that are accelerated towards each other. As they reach the center of the trap, they will collide with a velocity that is dependent on the shape of both the single- and double-well potential. As these shapes can be readily varied, the velocity of the molecules is tunable. Combining this feature with an inherently low velocity spread of the trapped molecules makes rapid single- to doublewell conversion an excellent starting point for studying collisions at low temperatures.

\section{THE VARIOUS TRAPPING CONFIGURATIONS}

In a cylindrically symmetric geometrv, the electric potential $\Phi(\rho, z)$ can be expressed as [14, 15, 20]:

$$
\Phi(\rho, z)=\Phi_{0}+\Phi_{1} \frac{z}{z_{0}}+\Phi_{2} \frac{\left(z^{2}-\rho^{2} / 2\right)}{z_{0}^{2}}+\Phi_{3} \frac{\left(z^{3}-3 \rho^{2} z / 2\right)}{z_{0}^{3}} \ldots,
$$

with $z_{0}$ a characteristic length scale. Here, the first term is a constant voltage, the second term a constant electric field, the third a quadrupolar electric field and the fourth a hexapolar electric field. A molecule with a quadratic Stark shift will experience a harmonic force 
in a quadrupolar electric field, whereas a hexapolar field is needed to form a harmonic trap for a molecule with a linear Stark shift. In our experiments we use ${ }^{15} \mathrm{ND}_{3}$ molecules in the low-field seeking hyperfine levels of the $|J, K\rangle=|1,1\rangle$ inversion doublet of the vibrational and electronic ground state. These molecules experience a quadratic Stark shift for small electric field strengths and a linear Stark shift for larger electric field strengths. The dependence of the Stark shift on the electric field strength $E$ for ${ }^{15} \mathrm{ND}_{3}$ in these levels is given by [21]:

$$
W_{\text {Stark }}(E)=\sqrt{\left(\frac{W_{i n v}}{2}\right)^{2}+\left(\frac{1}{2} \mu E\right)^{2}}-\left(\frac{W_{i n v}}{2}\right),
$$

with $W_{i n v}$ the inversion splitting and $\mu$ the dipole moment. For ${ }^{15} \mathrm{ND}_{3}$ the inversion splitting is $1430.3 \mathrm{MHz}$ 8] and the dipole moment is taken to be the same as for ${ }^{14} \mathrm{ND}_{3}$, i.e. $1.48 \mathrm{D}$ [22]. At even larger field strengths, the effect of mixing with higher rotational levels becomes more important and higher order terms have to be taken into account.

Using the four electrode geometry shown in Fig. 1 it is possible to create a field with dominant dipolar, quadrupolar or hexapolar contributions, by applying the appropriate voltages. The geometry of the trap is the same as was used previously to create an ac electric trap [23. It consists of two rings with an inner diameter of $10 \mathrm{~mm}$ and two endcaps. The two rings have a width of $4.6 \mathrm{~mm}$ and are separated by $2.9 \mathrm{~mm}$, whereas the two endcaps are separated by $2 z_{0}=9.1 \mathrm{~mm}$. They each have a hole with a diameter of $2.0 \mathrm{~mm}$, the first to allow molecules to enter and the second to extract the ions that are produced in the detection scheme.

In Fig. 2 the voltages needed for two different trapping fields are shown. When a voltage $-U_{2}$ is applied to the two ring electrodes and a voltage $U_{2}$ is applied to both endcaps, such as shown on the left-hand side of the figure, a field is formed with a dominant quadrupole term. Using a commercially available finite element program [24] the potential of this configuration is simulated. Below the trap cross-section the electric field strength as a function of position is shown for both the $\rho$-direction (solid line) and the z-direction (dotted line). Fitting the corresponding potential to equation 1 we find that $\Phi_{0} \approx-0.43 U_{2}$, $\Phi_{2} \approx 1.05 U_{2}, \Phi_{4} \approx 0.35 U_{2}$, and $\Phi_{6} \approx-0.04 U_{2}$ and that the uneven terms are zero due to symmetry. Beneath the electric field strength in Fig. 2] the Stark energy in mK ( $T=W_{\text {Stark }} / k$, with $k$ the Boltzmann constant) of a ${ }^{15} \mathrm{ND}_{3}$ molecule in this field is shown. The electric field in the quadrupole trap increases linearly with distance, but, because of the nonlinear dependence of the Stark energy on the electric field, the potential is harmonic near the center of the trap. Further away from the center the potential is linear. The resulting trap is tight (the trapping frequency is $\omega_{z} / 2 \pi=1.7 \mathrm{kHz}$ at the center of the trap) and has a depth of $0.09 \mathrm{~K}$ for ${ }^{15} \mathrm{ND}_{3}$.

A hexapole trap results when the voltages $U_{3}$ and $-U_{3}$ are applied alternately to the four electrodes of the trap as shown on the right-hand side of Fig. 2 A fit of the potential of this field to equation 1 yields $\Phi_{1}=0, \Phi_{3} \approx U_{3}$
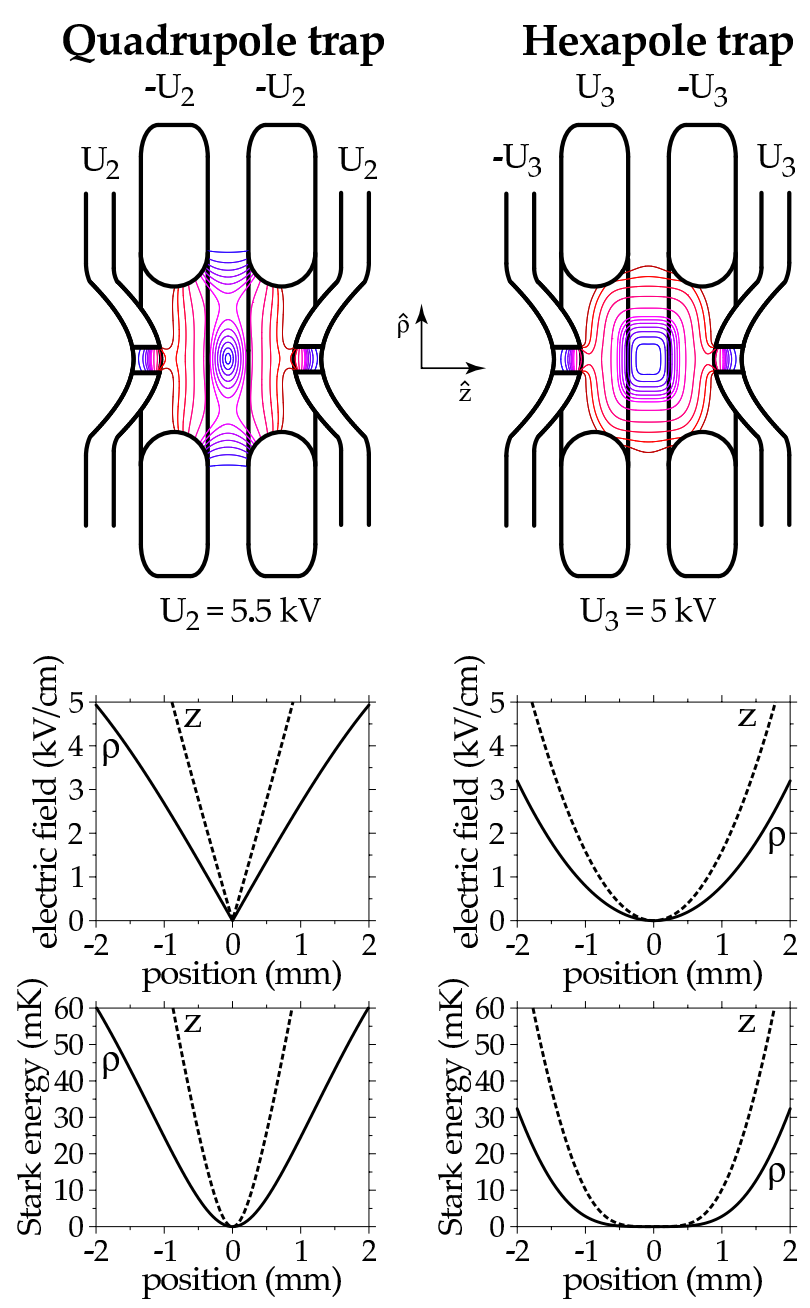

FIG. 2: Two cross-sections of the trap. On the left-hand side, the voltages that result in a quadrupole trap are shown, along with lines of constant electric field for electric field strengths of every $\mathrm{kV} / \mathrm{cm}$ from 1 to $7 \mathrm{kV} / \mathrm{cm}$ and every $5 \mathrm{kV} / \mathrm{cm}$ from 10 to $25 \mathrm{kV} / \mathrm{cm}$ (shown in color online, with increasing field strength going from blue to red). On the right-hand side, the same is shown for a hexapole trap. Below the two crosssections, the electric field is shown along with the Stark energy of a ${ }^{15} \mathrm{ND}_{3}$ molecule as a function of position in the $\rho$ - and z-direction (solid line and dotted line, respectively).

and $\Phi_{5} \approx-0.04 U_{3}$, with the even terms being zero due to symmetry. Again, both the electric field strength along the $\rho$ - and z-direction (solid and dotted line, respectively) and the Stark energy of a ${ }^{15} \mathrm{ND}_{3}$ molecule in this field are shown below the cross-sections of the trap. The electric field strength in this trap is quadratically dependent on the distance from the center. However, due to the nonlinear Stark effect the potential is essentially flat near the center of the trap. Away from the center the potential becomes harmonic. This trap is less tight (the trapping frequency is $\omega_{z} / 2 \pi=0$ at the center of the trap and goes to $\omega_{z} / 2 \pi=750 \mathrm{~Hz}$ further away from the center) and has a depth of $0.35 \mathrm{~K}$ for ${ }^{15} \mathrm{ND}_{3}$.

When applying voltages of $-U_{1},-0.6 U_{1}, 0.6 U_{1}$, and $U_{1}$ 


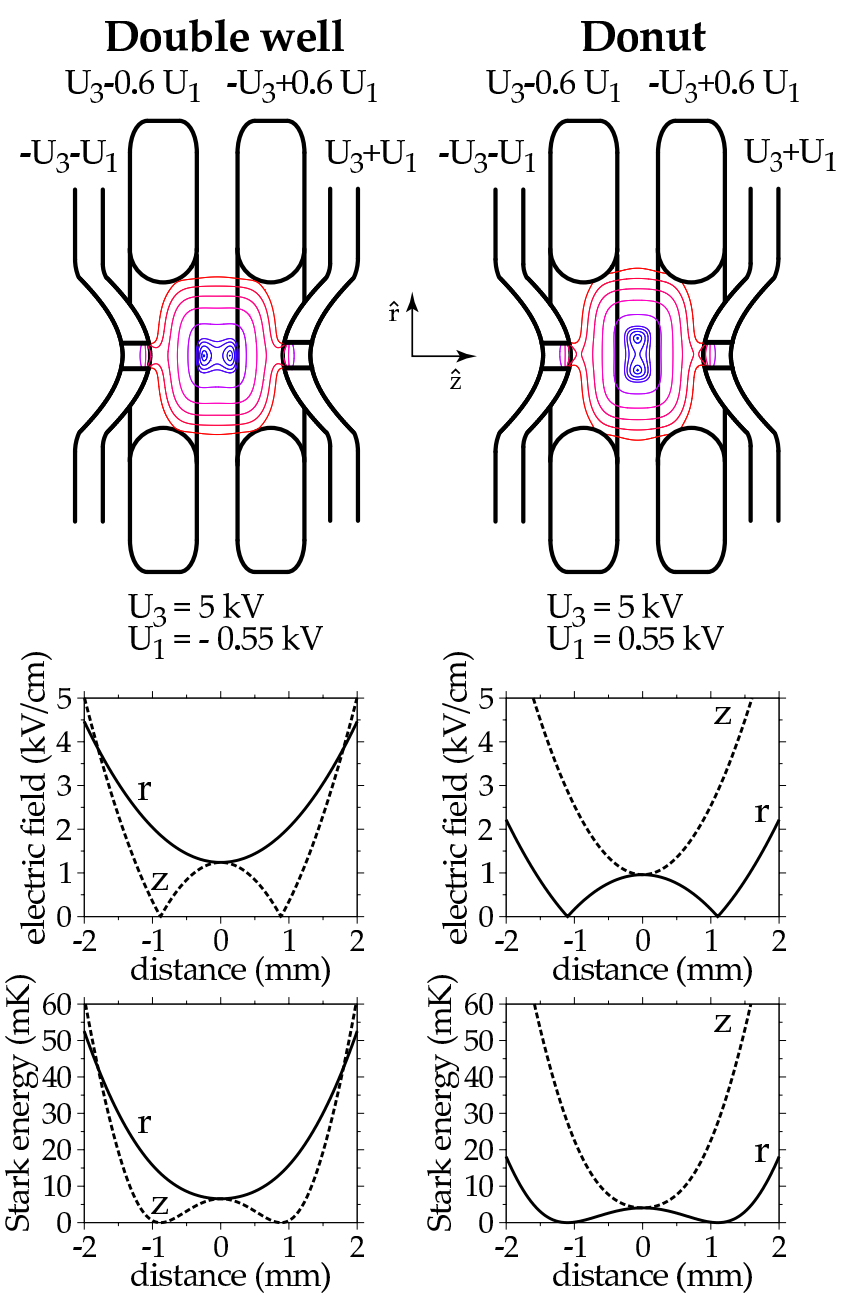

FIG. 3: Two cross-sections of the trap. On either side the voltages that are applied when a dipole field is added to a hexapole field are shown. Lines of constant electric field are shown for electric field strengths of $0.1 \mathrm{kV} / \mathrm{cm}$, every $0.5 \mathrm{kV} / \mathrm{cm}$ from 0.5 to $2 \mathrm{kV} / \mathrm{cm}$, and every $5 \mathrm{kV} / \mathrm{cm}$ from 5 to $25 \mathrm{kV} / \mathrm{cm}$ (shown in color online, with increasing field strength going from blue to red). On the left-hand side (righthand side) $U_{1}$ and $U_{3}$ have the same (opposite) sign. In each case both the electric field and the Stark energy of a ${ }^{15} \mathrm{ND}_{3}$ molecule as a function of position are shown in both the $\rho$ and z-direction (solid line and dotted line, respectively).

consecutively to the four electrodes of the trap, a dipolar field is formed. Fitting the potential of this field to equation 1 results in $\Phi_{1} \approx 0.88 U_{1}, \Phi_{3}=0$ and $\Phi_{5} \approx 0.09 U_{1}$, with the even terms being zero due to symmetry. Adding a dipole field to a hexapole field results in either a doublewell potential or a donut potential, depending on the relative sign of the dipole and hexapole term. In Fig. 3 both configurations are shown, along with the electric field strength and the Stark energy of a ${ }^{15} \mathrm{ND}_{3}$ molecule in this field along the $\rho$ - and z-direction (solid and dotted line, respectively). Both the distance between the two minima in the double-well trap and the diameter of the ring-shaped minimum in the donut trap are depen- dent on the ratio between the dipole and hexapole term. In the upper graph of Fig. [4 the distance between the two wells is shown as a function of the ratio $\left|U_{1} / U_{3}\right|$ in the case of an ideal hexapole and dipole field (solid line). The dotted line shows the same for the diameter of the donutshaped minimum. The crosses and triangles (double-well trap and donut trap, respectively) indicate the simulated value of this well separation, using the finite element program with $U_{3}=5 \mathrm{kV}$ and different values of $U_{1}$.

The height of the barrier between the two wells is shown in the lower graph of Fig. [4 given in $\mathrm{mK}$ for ${ }^{15} \mathrm{ND}_{3}$. In the case of an ideal hexapole and dipole field (solid line), this height is the same for the double-well and donut trap and is solely dependent on the value of $\left|U_{1}\right|$. Note that the barrier height is identical to the amount of potential energy gained by a ${ }^{15} \mathrm{ND}_{3}$ molecule in the center of one of the wells when switching to the singlewell configuration. This can be seen by realizing that in the center of the double-well trap, i.e. at the top of the barrier, the electric field strength is determined by the dipole term only, as the hexapole field is zero there. In the center of one of the wells the hexapole field is equal to the dipole field, although opposite in direction. Therefore, when switching the dipole field off to produce a single-well trap, molecules originally at the center of one of the two wells will now have a potential energy equal to the barrier height. Values for the barrier height were obtained using the finite element program as well and are shown as crosses (double-well trap) and triangles (donut trap) in the lower graph of Fig. 4 From these two graphs it is clear that both the well separation and the barrier height, and therefore also the collision energy of the molecules upon double- to single-well conversion, can easily be changed by varying the voltages on the four electrodes. At the maximum value of $\left|U_{1}\right|$ used in the experiment, $\left|U_{1}\right|=0.55 \mathrm{kV}$, the barrier height is on the order of $5 \mathrm{mK}$. The separation between the wells is $1.8 \mathrm{~mm}$ in this case $\left(U_{3}=5 \mathrm{kV}\right)$, whereas the diameter of the donut-shaped minimum is $2.2 \mathrm{~mm}$.

\section{EXPERIMENT}

In Fig. 5 the experimental setup is shown. In the experiments, the trap is loaded from a Stark-decelerated molecular beam. A mixture of $5 \%{ }^{15} \mathrm{ND}_{3}$ seeded in Xenon is supersonically expanded from a pulsed valve at a $10 \mathrm{~Hz}$ rate. Due to cooling of the valve to $-70^{\circ} \mathrm{C}$, the gas pulse has a velocity of $280 \mathrm{~m} / \mathrm{s}$. In the expansion, about $60 \%$ of the ammonia molecules are internally cooled to the ground state of para-ammonia, the $|J, K\rangle=|1,1\rangle$ inversion doublet in the electronic and vibrational ground state. After passing through a skimmer, those molecules that reside in the low-field seeking hyperfine levels of the upper component of the inversion doublet are focused into the Stark decelerator by a $6 \mathrm{~cm}$ long hexapole. Molecules in high-field seeking levels are attracted by the high electric fields of both the 

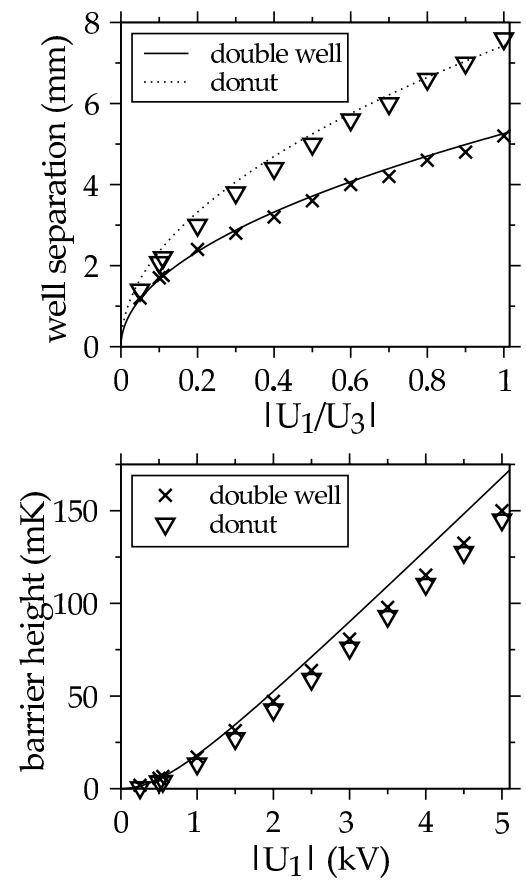

FIG. 4: Well separation as a function of $\left|U_{1} / U_{3}\right|$ and barrier height (for ${ }^{15} \mathrm{ND}_{3}$ ) as a function of $\left|U_{1}\right|$ in the ideal doublewell (solid line) and donut trap (dotted line). In both graphs the crosses (double-well trap) and triangles (donut trap) show the same quantities obtained from electric field simulations of the actual trap geometry.

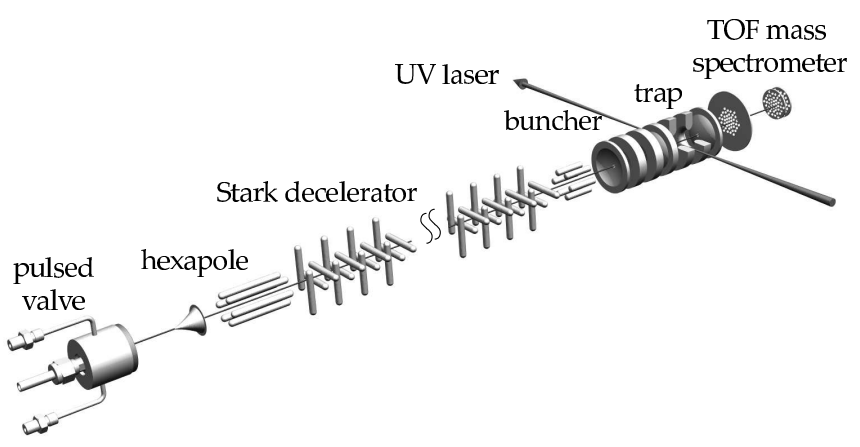

FIG. 5: Experimental setup. A pulsed supersonic beam of ${ }^{15} \mathrm{ND}_{3}$ molecules seeded in Xenon exits a cooled valve with a velocity of $280 \mathrm{~m} / \mathrm{s}$. Ammonia molecules in the low-field seeking hyperfine levels of the $|J, K\rangle=|1,1\rangle$ state are focused into the decelerator by a hexapole, decelerated, and then transversally and longitudinally focused into the trap by a hexapole and buncher, respectively. The molecules are detected in a TOF-mass spectrometer.

hexapoles and the decelerator and will be lost from the beam. Molecules that reside in the remainder of the hyperfine levels of the inversion doublet, that only have a higher order Stark effect, will hardly be effected by the electric fields. The decelerator consists of 95 pairs of parallel $3 \mathrm{~mm}$-diameter rods, with a closest distance of $2 \mathrm{~mm}$ between two rods and a closest distance of $2.5 \mathrm{~mm}$ between pairs of rods. Each consecutive pair of rods is rotated over $90^{\circ}$ and within each pair, a positive voltage of $10 \mathrm{kV}$ is applied to one electrode, whereas the same negative voltage is applied to the other. A more extensive description of this part of the setup, along with the operation principle of the decelerator can be found elsewhere [8, 25]. Using a phase angle of $57.5^{\circ}$ a subset of lowfield seeking molecules with an initial velocity of around $280 \mathrm{~m} / \mathrm{s}$ is decelerated to about $15 \mathrm{~m} / \mathrm{s}$ at the exit of the decelerator. After $5 \mathrm{~mm}$ the molecules are transversally focused into the trap by a second (12.5 $\mathrm{mm}$ long) hexapole. Subsequently, they are longitudinally focused into the trap using a buncher that is placed another $5 \mathrm{~mm}$ downstream of the hexapole. The buncher has a similar geometry as the trap. Before the ammonia molecules enter the trap, voltages are applied to the trap such that a last electric field slope is formed. In gaining Stark energy when entering the trap, the low-field seeking molecules lose their last kinetic energy and reach a stand-still in the middle of the trap. At this moment the voltages on the four electrodes are switched to generate a particular trapping potential, thereby confining the molecules in either a quadrupole, hexapole, double-well or donut potential. After a certain trapping time, the trap is turned off and molecules in the upper component of the inversion doublet are ionized in a state-selective $(2+1)$-Resonance Enhanced Multi Photon Ionization (REMPI) scheme, using a pulsed UV-laser around $317 \mathrm{~nm}$ [26]. The resulting ions are subsequently detected in a time-of-flight (TOF) mass-spectrometer. Using a $75 \mathrm{~cm}$ lens the ionization laser is focused into the trap passing through the $2.9 \mathrm{~mm}$ gap between the two ring electrodes. The laser focus is estimated to be about $200 \mu \mathrm{m}$ in diameter. Applying small bias voltages of $200 \mathrm{~V}, 150 \mathrm{~V},-150 \mathrm{~V}$ and $-200 \mathrm{~V}$ to the four electrodes of the trap results in an electric field that accelerates the ions towards the detector. The ions are detected over a length of $2 \mathrm{~mm}$ along the laserbeam, limited by the size of the hole in the endcap. Using a computer controlled translation stage, the focus of the laserbeam can be moved along the beam-axis, i.e. along the z-direction, over about $2.5 \mathrm{~mm}$. In this way, the spatial distribution of the trapped molecules can be measured.

\section{RESULTS AND DISCUSSION}

In Fig. 6, the density of trapped ${ }^{15} \mathrm{ND}_{3}$ molecules is shown as a function of position in the z-direction, both for hexapole and for quadrupole trapping voltages. The measurements are taken after the molecules have been trapped for about $72 \mathrm{~ms}$. We found that in order to obtain a single-well profile for the molecules confined in the hexapole trap a small dipole term had to be added. We attribute this to deviations of the distances between the electrodes from the ideal geometry. This compensating dipole term is added in all subsequent measurements. As the quadrupole trap is much tighter than the 


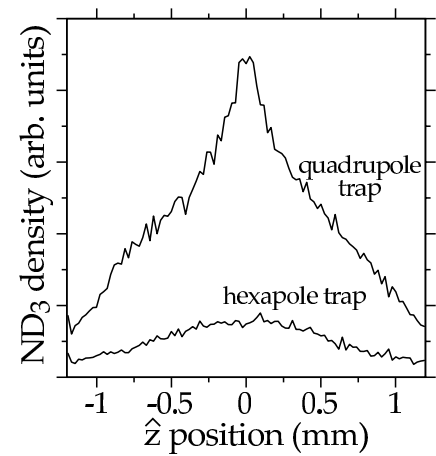

FIG. 6: Density of trapped ${ }^{15} \mathrm{ND}_{3}$ molecules as a function of position in the z-direction for both the quadrupole trap (upper trace) and the hexapole trap (lower trace), after trapping for about $72 \mathrm{~ms}$.

hexapole trap (see Fig. 2), a signal difference arises between the two traces. The peak density of molecules in the hexapole trap is about $10^{7} \mathrm{~cm}^{-3}$, whereas it is about ten times higher in the quadrupole trap. The loading process is identical for both traps and is matched to the hexapole trap, resulting in a less than perfect match of the decelerated packet to the quadrupole trap. This gives rise to the peculiar shape of the measured distribution in the quadrupole trap, which seems to consist of two packets with different temperatures.

In Fig. 7 the density of ${ }^{15} \mathrm{ND}_{3}$ molecules in the hexapole trap is shown as a function of position in the z-direction. By adding a dipole term to the hexapole term, the shape of the trap and thereby the profile of the trapped molecules can be changed significantly. Each trace in Fig. 7 corresponds to a different value of the added dipole term. When no dipole is added, the trap consists of only a single well. A single peak results, as trace (d) shows. Adding a negative dipole term transforms the single well to a double well, with a distance and barrier between the two wells that depend on the value of the dipole term. The upper three traces of Fig. [ 7 show two peaks that move closer together as the dipole term is increased from $-0.55 \mathrm{kV}$ (trace (a)) to $-0.3 \mathrm{kV}$ (trace (b)) to $-0.15 \mathrm{kV}$ (trace (c)). When a positive dipole term is added, the shape of the trap becomes that of a donut. As only molecules in the relatively small laser focus near the center of the trap are ionized in the detection scheme, this transformation to a donut-shaped trap leads to a reduction in signal only, as is shown by trace (e) and (f) ( $U_{1}=0.25 \mathrm{kV}$ and $U_{1}=0.55 \mathrm{kV}$, respectively).

At the densities that we have in the trap, no thermalizing collisions occur, and formally no temperature can be assigned. To nevertheless characterize the trapped sample in terms of a temperature, we fitted the density profile to the thermal density distribution [27]:

$$
n(\rho, z)=n_{0} e^{-W_{\text {Stark }}(\rho, z) / k T},
$$

with $n_{0}$ the density at the trap minimum and $W_{\text {Stark }}(\rho, z)$ the potential energy as a function of posi-
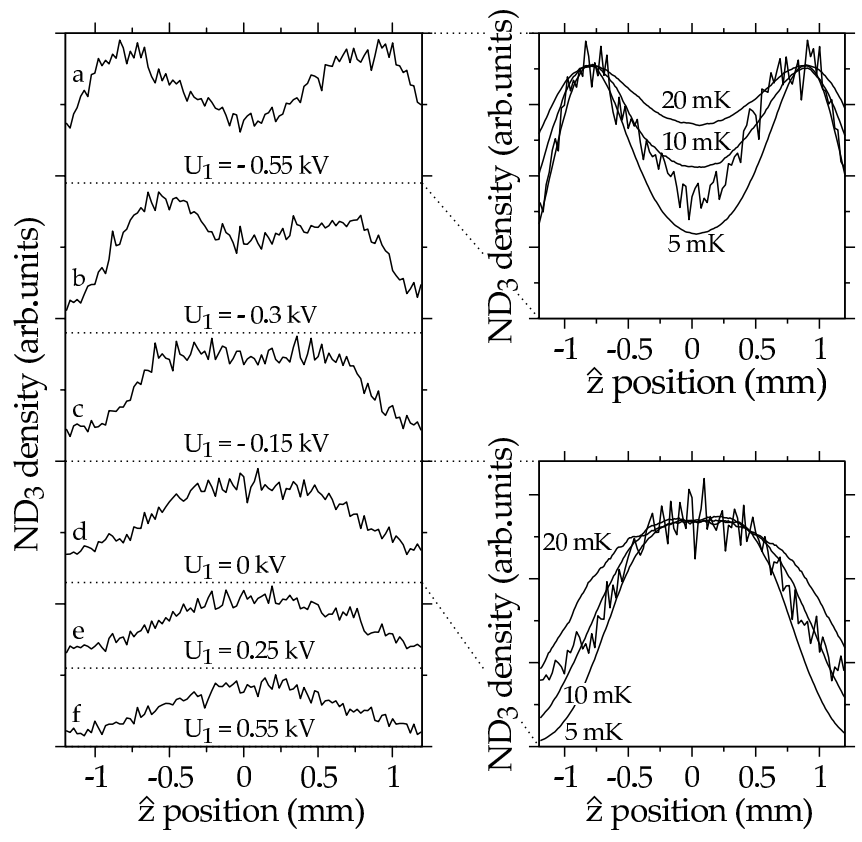

FIG. 7: Density of ${ }^{15} \mathrm{ND}_{3}$ molecules confined in a hexapole trap as a function of position in the z-direction. A negative dipole term of $-0.55 \mathrm{kV},-0.3 \mathrm{kV}$ and $-0.15 \mathrm{k} \mathrm{V}$ is added to the hexapole term $\left(U_{3}=5 \mathrm{kV}\right)$ in the upper three traces (a-c), resulting in a double-well potential. Trace (d) shows a packet in a purely single well. A positive dipole term of $0.25 \mathrm{kV}$ and $0.55 \mathrm{kV}$ is added for the lower two traces (e-f), thereby creating a donut potential. On the right-hand side, trace (a) and (d) are shown once more, along with simulations of the density distribution of the packet of molecules for temperatures of 5,10 and $20 \mathrm{mK}$.

tion. On the right-hand side of Fig. 7 the measurements with a dipole term of $U_{1}=-0.55 \mathrm{kV}$ and $U_{1}=0 \mathrm{kV}$ are shown once more, along with the thermal density distribution as obtained from equation (3) for temperatures of 5, 10 and $20 \mathrm{mK}$. It can be seen that in either case the simulated distribution with a temperature of $10 \mathrm{mK}$ matches the measurement best. The same simulation has been performed for the spatial distribution of molecules confined in a quadrupole trap. Due to the seemingly double profile, it is more difficult to describe this distribution with a single temperature. A temperature of $75 \mathrm{mK}$ seems to best describe the broad base, whereas the sharp center peak agrees best with a temperature of $5 \mathrm{mK}$. An alternative method to deduce a temperature is to measure the width of the packet of trapped molecules as a function of time after the trap has been turned off. Measurements of this type are shown in Fig. 8. On the left-hand side two profiles of ${ }^{15} \mathrm{ND}_{3}$ molecules are seen, one after $20 \mu$ s and one after $420 \mu$ s after switching off the hexapole trap (upper and lower curve, respectively). In either case the molecules have been confined for about $72 \mathrm{~ms}$. Due to the velocity spread of the molecules the packet spreads out after the trap has been turned off, resulting in less signal 

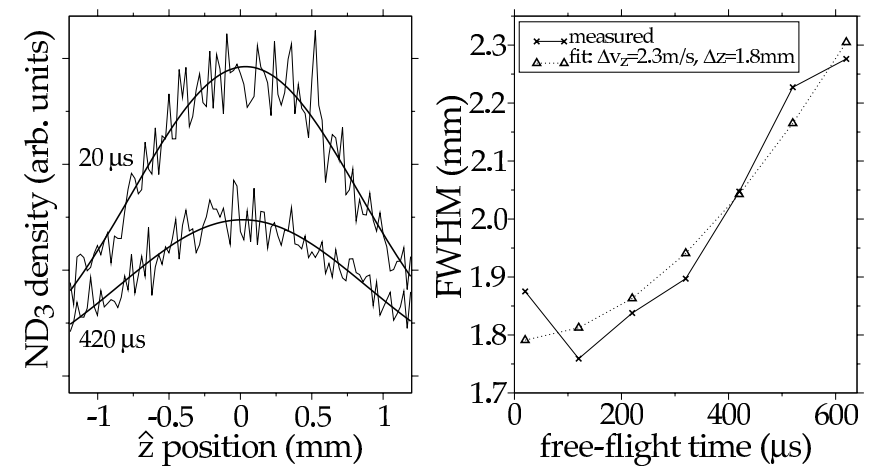

FIG. 8: Determination of the temperature of a packet of ${ }^{15} \mathrm{ND}_{3}$ molecules trapped in the hexapole trap. On the left, the profile of a packet is shown $20 \mu$ s and $420 \mu$ s after the trap is turned off (upper and lower trace, respectively). From a fit to a Gaussian lineshape the FWHM of the distribution is obtained, as shown as a function of free-flight time (solid line with crosses) on the right-hand side of the figure.

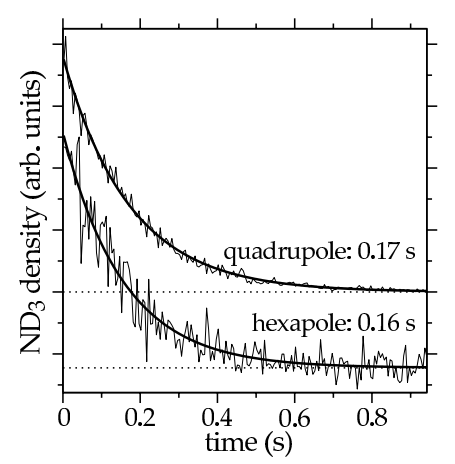

FIG. 9: Density of ${ }^{15} \mathrm{ND}_{3}$ molecules in the quadrupole (upper trace) and the hexapole (lower trace) trap as a function of time that the trap is on.

and an increased width. To obtain this width a Gaussian lineshape is fitted to each profile. The acquired full-width-half-maximum (FWHM) is shown as a function of free-flight time on the right-hand side of the figure (solid line with crosses). To deduce a temperature, this curve is fitted to $\sqrt{(\Delta z)^{2}+\left(\Delta v_{z} \cdot t\right)^{2}}$ (dotted line with triangles), resulting in a velocity spread of $2.3 \mathrm{~m} / \mathrm{s}$ and a position spread of $1.8 \mathrm{~mm}$ (both FWHM). Using $\Delta v_{z}=\sqrt{8 \ln 2} \cdot \sqrt{k T / m}[28]$, this gives a temperature of about $2.5 \mathrm{mK}$.

The different temperatures of $10 \mathrm{mK}$ and $2.5 \mathrm{mK}$ obtained by the two different methods actually correspond to a difference of only a few tenths of a $\mathrm{mm}$ in the width of the initial packet. Such a small difference can be explained by possible misalignments of the electrodes of the trap, causing uncertainties in the actual electric field, and therefore in $W_{\text {Stark }}(\rho, z)$. Moreover, extracting the width of the packets from the measurements is quite sensitive to how the baseline is taken into account in the fit. All together, this analysis only serves to indicate that the temperature of the trapped sample is in the $\mathrm{mK}$ range.

In Fig. 9 the density of ammonia molecules is shown in both the quadrupole and the hexapole trap as a function of time that the trap is on. The molecules are seen to leave the trap with a $1 / e$ time of $0.16 \mathrm{~s}$ for the hexapole trap and $0.17 \mathrm{~s}$ for the quadrupole trap, the same within error bars. The trapping lifetime is mainly limited by background collisions (background pressure $4 \cdot 10^{-8}$ mbar). Optical pumping by black-body radiation cannot be completely neglected, however. In the roomtemperature trap, pumping of the ${ }^{15} \mathrm{ND}_{3}$ molecules to the vibrationally excited $\nu_{2}$ level (umbrella mode) around $750 \mathrm{~cm}^{-1}$ is expected to occur on the time-scale of a second.

\section{CONCLUSIONS}

We have presented a new electrostatic trapping geometry, that illustrates the versatility of electric fields in forming tailored trapping potentials. By applying different voltages to a set of four electrodes, different trapping fields are created, between which rapid switching on a timescale of $100 \mathrm{~ns}$ is possible. Spatial distributions and lifetimes of a cold packet of ${ }^{15} \mathrm{ND}_{3}$ molecules loaded into both a hexapole and a quadrupole trap are shown. Additionally, we studied a hexapole trap that transforms into a double-well or donut trap when different dipole terms are added. The barrier height and well separation of either one of these two traps depend on the strength of the dipolar and hexapolar electric field and are easily changed.

Rapid switching between, for instance, the double-well trap and a pure hexapole trap offers good prospects for measuring collision cross-sections as a function of collision energy at low temperatures. The amount of energy gained by molecules trapped in one of the wells after switching to the single-well depends solely on the strength of the electric dipole field, which can be easily varied.

\section{Acknowledgments}

This work is part of the research program of the 'Stichting voor Fundamenteel Onderzoek der Materie (FOM)', which is financially supported by the Netherlands Organisation for Scientific Research (NWO). H.L.B. acknowledges financial support from NWO via a VENI-grant. The research of M.S. was made possible through a Liebig stipend of the 'Fonds der Chemischen Industrie'. We thank André J.A. van Roij and Henrik Haak for the mechanical design and construction of the trap and Boris Sartakov for helpful discussions. 
[1] K. Burnett, P. S. Julienne, P. D. Lett, E. Teisinga, and C. J. Williams, Nature 416, 225 (2002).

[2] N. Balakrishnan and A. Dalgarno, J. Chem. Phys. 113, 621 (2000).

[3] N. Balakrishnan and A. Dalgarno, Chem. Phys. Lett. 341, 652 (2001).

[4] J. L. Bohn, Phys. Rev. A 63, 052714 (2001).

[5] L. Santos, G. V. Shlyapnikov, P. Zoller, and M. Lewenstein, Phys. Rev. Lett. 85, 1791 (2000).

[6] K. Góral, L. Santos, and M. Lewenstein, Phys. Rev. Lett. 88, 170406 (2002).

[7] J. Stuhler, A. Griesmaier, T. Koch, M. Fattori, T. Pfau, S. Giovanazzi, P. Pedri, and L. Santos, Phys. Rev. Lett. 95, 150406 (2005).

[8] J. van Veldhoven, J. Küpper, H. L. Bethlem, B. Sartakov, A. J. van Roij, and G. Meijer, Eur. Phys. J. D 31, 337 (2004).

[9] E. R. Hudson, H. J. Lewandowski, B. C. Sawyer, and J. Ye, arXiv p. physics/0601054 (2006).

[10] W. H. Wing, Phys. Rev. Lett. 45, 631 (1980).

[11] H. L. Bethlem, G. Berden, F. M. H. Crompvoets, R. T. Jongma, A. J. A. van Roij, and G. Meijer, Nature 406, 491 (2000).

[12] F. M. H. Crompvoets, H. L. Bethlem, R. T. Jongma, and G. Meijer, Nature 411, 174 (2001).

[13] S. Y. T. van de Meerakker, P. H. M. Smeets, N. Vanhaecke, R. T. Jongma, and G. Meijer, Phys. Rev. Lett. 94, 023004 (2005).

[14] N. E. Shafer-Ray, K. A. Milton, B. R. Furneaux, E. R. I. Abraham, and G. R. Kalbfleisch, Phys. Rev. A 67, 045401 (2003).

[15] G. Xu, Ph.D. thesis, The University of Texas at Austin (2001).
[16] M. R. Andrews, C. G. Townsend, H.-J. Miesner, D. S. Durfee, D. M. Kurn, and W. Ketterle, Science 275, 637 (1997).

[17] S. Inouye, S. Gupta, T. Rosenband, A. P. Chikkatur, A. Görlitz, T. L. Gustavson, A. E. Leanhardt, D. E. Pritchard, and W. Ketterle, Phys. Rev. Lett. 87, 080402 (2001).

[18] M. Albiez, R. Gati, J. Fölling, S. Hunsmann, M. Cristiani, and M. K. Oberthaler, Phys. Rev. Lett. 95, 010402 (2005).

[19] C. Buggle, J. Léonard, W. von Klitzing, and J. T. M. Walraven, Phys. Rev. Lett. 93, 173202 (2004).

[20] T. Bergeman, G. Erez, and H. J. Metcalf, Phys. Rev. A 35, 1535 (1987).

[21] C. H. Townes and A. L. Schawlow, Microwave Spectroscopy (Dover Publications, New York, 1975).

[22] R. L. Bhattacharjee, L. H. Johnston, G. R. Sudhakaran, and J. C. Sarker, J. Mol. Spec. 138, 38 (1989).

[23] J. van Veldhoven, H. L. Bethlem, and G. Meijer, Phys. Rev. Lett. 94, 083001 (2005).

[24] D. A. Dahl, Simion 3D Version 6.0 (Idaho National Engineering Laboratory, Idaho Falls (USA), 1995).

[25] H. L. Bethlem, F. M. H. Crompvoets, R. T. Jongma, S. Y. T. van de Meerakker, and G. Meijer, Phys. Rev. A 65, 053416 (2002).

[26] M. N. R. Ashfold, R. N. Dixon, N. Little, R. J. Stickland, and C. M. Western, J. Chem. Phys. 89, 1754 (1988).

[27] O. J. Luiten, M. W. Reynolds, and J. T. M. Walraven, Phys. Rev. A 53, 381 (1996).

[28] F. Reif, Fundamentals of statistical and thermal physics (McGraw-Hill, Singapore, 1985). 